\title{
Impact of chirality in the amorphous state of conglomerate forming systems: case study of $\mathrm{N}$-acetyl- $\alpha$-methylbenzylamine
}

\section{Authorship:}

Bienvenu Atawa $\uparrow \S T^{*}$, Nicolas Couvrat $\S$, Frédéric Affouard‡, Natália T. Correiaキ, Gérard Coquerel §, Allisson Saiter-Fourcin †

† Normandie Univ, UNIROUEN, INSA Rouen, CNRS, GPM, 76000 Rouen, France

$\S$ Normandie Univ, UNIROUEN, SMS, 76000 Rouen, France

f Univ Lille, CNRS, INRA, ENSCL, UMR 8207, UMET, Unité Matériaux et Transformations, F59000 Lille, France

T Univ-Lyon, Université Lyon 1, Ingénierie des Matériaux Polymères, CNRS UMR 5223, 69622

Villeurbanne, France

\begin{abstract}
:
The present work aims at addressing the issue of molecular handedness in the glassy and liquid states and its impact on heterogeneous equilibrium. For this purpose we evaluated the glass forming ability (GFA), the crystallization propensity, the molecular mobility and the hydrogen bonding structure of a chiral conglomerate forming system $\mathrm{N}$-acetyl- $\alpha$-methylbenzylamine (Nac-MBA) at various enantiomeric excess (ee) by mean of experimental and computational techniques. We revealed that the rich relaxational landscape (Debye (D), $\alpha, \beta_{\mathrm{JG}}$ and $\gamma$ ) and the temperature dependence of the time scale of each process were insensitive to chirality. The most remarkable impact of chirality was expressed on the GFA and the recrystallization of heterochiral arrangements. In fact the GFA increases with decreasing ee while the crystallization propensity increases with increasing ee. The counter enantiomer acts as a disruptor of crystallization and favored the glass formation upon cooling. The Molecular Dynamic Simulation (MDS) results on the architecture of chiral sequences showed that homochiral sequences were most favorable when compared to heterochiral in the liquid state. However, this predisposition to form homochiral sequences in the liquid state was not precursor of the future crystalline structure since the liquid or the glassy system recrystallizes as heterochiral sequences. At our understanding the crystallization was mostly
\end{abstract}


controlled by the mean free migration path of an enantiomer to build homochiral or heterochiral sequences. In the present case, it seems that the mean free migration path achieved by an enantiomer for heterochiral sequences is lower compared to homochiral arrangements in such a way that the crystallization of the metastable racemic compound is kinetically more favorable.

\section{Introduction}

Chirality as well as its stereochemical, structural and thermodynamic implications, play an important role in many biological and physicochemical processes ${ }^{1}$. Additionally, chirality is of primary importance in advance photonic applications ${ }^{2,3}$ (such as alternative routes to negative refraction), in low-power display technologies ${ }^{4,5}$ and in the processing of pharmaceutical drugs ${ }^{1,6,7}$. Indeed more than $50 \%$ of pharmaceutical marketed drugs are chiral ${ }^{1}$. The concept of chirality derived historically from the distinction between the configurational isomers of asymmetric molecules discovered by Pasteur ${ }^{8}$. Configurational isomers, also termed optical or chiral isomers, can be classified as R or Senantiomers. $\mathrm{R}$ and $\mathrm{S}$-enantiomers are pairs of configurational isomers that are mirror images of each other and are non-superimposable. They do possess identical scalar properties in achiral environments but opposite vector properties ${ }^{7,9}$. Since the thalidomide tragedy, it is well establish that opposite enantiomers often differ significantly in their pharmacological, toxicological, pharmacodynamics and pharmacokinetic properties ${ }^{1}$. Indeed the pharmaceutical industry pursues the development of design principles for asymmetric synthesis ${ }^{1,10}$ or efficient separation ${ }^{11}$ of chirality-tailored compounds. Pure enantiomers differ in their properties from racemic species since the interactions between homochiral molecules are typically different from heterochiral arrangements. Starting from a solution of a racemic mixture (i.e. containing an equimolar mixture between the two optical antipodes), several crystalline packing are possible during crystallization: i) a homo-chiral stacking leading to a conglomerate (equimolar eutectic mixture, about $5 \%$ of occurrence) or ii) a hetero-chiral stacking leading either to a racemic compound (cocrystal containing equal numbers of molecules of the both enantiomers representing 90 to $95 \%$ of racemic species) or solid solution containing equal quantities of both enantiomers randomly distributed in the crystal lattice (less than $1 \%)^{12}$.

A strong interest has been paid on the amorphous state of molecular compounds with therapeutical properties regarding the improved bioavailability compared to their crystalline counterpart ${ }^{13,14}$. Systematic studies on the amorphous state of molecular compounds with a stereogenic chiral center are largely available in the literature ${ }^{15-25}$. A recent and increasing interest is paid to the investigation of chirality influence in molecular amorphous systems regarding their molecular mobility ${ }^{26-29}$, their 
glass forming ability ${ }^{30}$ and their crystallization behavior ${ }^{26,28,31-33}$ as function of the initial enantiomeric composition. From these studies, it is clear that the glass transition temperature $\mathrm{Tg}$ appears to be independent of the enantiomeric excess (ee: the absolute difference between the mole fractions of each enantiomer (ee $=0 \%$ racemic composition, ee $=100 \%$ pure enantiomer) ${ }^{7,26-}$ $28,32,33$, same for the characteristic size of cooperative movements and relaxation landscape ${ }^{28,33}$. When differences exist, they are mostly expressed in the glass forming ability ${ }^{30}$ and the kinetic of crystallization ${ }^{26,30,33}$, structural effects due to specific interactions inducing changes in polarization and dielectric properties ${ }^{28,34}$. A brief picture of the state of the art related to the impact of chirality on the amorphous state is mentioned in one of our recent work ${ }^{28}$. Most of these studies are related to chiral species forming stable racemic compounds (intermediate compound between both enantiomers with an equimolar composition) ${ }^{26-29,32,33}$. The appearance of this defined compound in the binary system of both enantiomers could thus influence the relaxation and the crystallization phenomena additionally to chirality itself. In this work, in order to avoid such additional factors, we focused on an enantiomeric system forming a stable conglomerate at room temperature (full chiral discrimination in the solid state): namely $\mathrm{N}$-acetyl- $\alpha$-methylbenzylamine. In such cases, the counter enantiomer acts as an impurity (of the same chemical nature) until reaching a maximum of $50 \%$ (racemic composition) in such a way that no subsidiary effects of an intermediate compound unless chirality itself could influence the recrystallization behavior and the relaxation phenomena. Furthermore, this model system offers a rich relaxation landscape with four processes having different "microscopic origins" going from a slow Debye-like process to the fast secondary $\curlyvee$-process 35. The temperature dependence of their relaxation time is investigated as a function of the enantiomeric ratio. This sheds light on the effect of chirality on molecular mobility. Moreover the issue regarding the dynamical properties and the structure of homochiral and heterochiral arrangements in the liquid state is addressed by mean of Molecular Dynamic Simulations (MDS) Thus, this study focuses on the glass forming ability, the recrystallization tendency, and the phenomena of relaxation at several enantiomeric excess (ee) of Nac-MBA. In order to address some responses on the impact of chirality in the amorphous state and the competition between homochiral and heterochiral arrangements, an experimental and computational study of Nac-MBA system was performed by means of calorimetric techniques (such as Differential Scanning Calorimetry (DSC) Fast Scanning Calorimetry (FSC), Temperature Modulated DSC (MT-DSC)), structural characterization (Temperature Resolved X-ray diffraction), Dielectric Relaxation Spectroscopy (DRS) and MDS. 


\section{Experimental and Simulation}

Optically pure enantiomers $\mathrm{R}(+)$ and $\mathrm{S}(-)$ Nac-MBA are obtained from synthesis by an acetylation reaction of $R(+)$ and $S(-) \alpha$-methylbenzylamine with acetyl chloride. Both chemicals purchased from Sigma Aldrich. The synthesis and purification procedures were already detailed in our previous works ${ }^{31,35}$. All the analyzed compositions where prepared by mixing $\mathrm{R}(+)$ and $\mathrm{S}(-)$ enantiomers in order to obtain different ee

\subsection{Thermal characterization by classical DSC, MT-DSC and FSC measurements.}

DSC and MT-DSC measurements were performed with a Q100 DSC from TA Instruments equipped with a cooling system. The calibration steps have been explicitly detailed in our previous work ${ }^{35}$. All measurements were performed under inert nitrogen gas flow of $50 \mathrm{~mL} / \mathrm{min}$ with $5 \mathrm{mg}$ of sample obtained by mixing both enantiomers and encapsulated in a $T_{\text {zero }}$ aluminium pan. The conventional DSC measurements where performed at a heating rate of $10 \mathrm{~K} / \mathrm{min}$ and the MT-DSC experiments were carried out in the "heat only" modulation mode with an oscillation amplitude of $( \pm) 0.318 \mathrm{~K}$ and oscillation period of $60 \mathrm{~s}$, during heating at $2 \mathrm{~K} / \mathrm{min}$, the best protocol to explore crystallization/melting processes. The melted sample was initially cooled at an average cooling rate of $30 \mathrm{~K} / \mathrm{min} 388 \mathrm{~K}$ down to $203 \mathrm{~K}$.

The FSC analyses were carried out with a power compensation twin-chip namely Flash DSC 1 from Mettler Toledo and equipped with a Huber TC 100 intra cooler. The calibration of the FSC consists of conditioning and correction procedures according to the manufacturer and prior to measurement. The powder sample was submitted to a nitrogen purge gas flow at a rate of $20 \mathrm{~mL} / \mathrm{min}$ and placed on the sample chip area ( $0.5 \mathrm{~mm}$ of diameter) by the mean of an optical microscope and a long hair fiber for sample positioning. The sample is then submitted to a heat/cool/heat process at 30000 $\mathrm{K} / \mathrm{min}$.

\subsection{Temperature Resolved X-ray Diffraction (TR-XRD)}

Powder x-ray diffraction data were recorded on a model D5005 diffractometer (Siemens-Bruker). The X-Ray tube is equipped with a copper anticathode and a $k \beta$ filter (Ni). The analysis step was set at $0.08^{\circ}$ with a counting time of $4 \mathrm{~s} /$ step in the range 3 to $30^{\circ}(2 \theta)$. These analyzes were resolved in temperature by means of a heating plate TTK 450 (Anton Paar) coupled with a Lauda RP890 intracooler for low temperatures. At each temperature, the analysis lasted 22.5 minutes and the heating rate between each temperature step was fixed at $2 \mathrm{~K} / \mathrm{min}$.

\subsection{Dielectric Relaxation Spectroscopy}


a) Low frequency range $10^{-1} \mathrm{~Hz}-2 \mathrm{MHz}$

The real and imaginary parts of the complex permittivity were recorded over the range $10^{-1} \mathrm{~Hz}-2$ $\mathrm{MHz}$ using Novocontrol Technologies $\mathrm{GmbH}$ Alpha dielectric spectrometer. The sample preparation and calibration of the equipment were previously reported in our previous work ${ }^{35}$.

Dielectric measurements were carried out in both isothermal and isochronal modes. In order to study the phase transitions in Nac-MBA system at several ee $(0 \%, 50 \%, 75 \%$ and $100 \%)$, the complex dielectric permittivity was measured in the isochronal mode, at frequencies high enough to allow a reliable measurement $\left(10^{2} \mathrm{~Hz}-2 \mathrm{MHz}\right)$ during heating/cooling cycles performed at a rate set to 1 $\mathrm{K} / \mathrm{min}$ in the temperature range between $223 \mathrm{~K}$ and $383 \mathrm{~K}$. For the isothermal measurements, the melt was promptly quenched in liquid nitrogen in order to ensure total amorphization. The dielectric spectra were collected isothermally from $153 \mathrm{~K}$ up to $273 \mathrm{~K}$ with a suitable consecutive step. Since crystallization occurs in the supercooled liquid region at all the analyzed compositions, high frequency measurements were also achieved upon melt cooling for frequency higher than $2 \mathrm{MHz}$.

b) High frequency range $1 \mathrm{MHz}-3 \mathrm{GHz}$

Measurements were performed in the range $1 \mathrm{MHz}-3 \mathrm{GHz}$ by means of a coaxial line reflectometer based on the impedance analyzer HP 4191 from Novocontrol Technologies GmbH. Prior to measurements, two successive calibration steps were achieved: Line and sample cell calibrations. Samples were prepared in parallel plate geometry between two gold-plated electrodes of $10 \mathrm{~mm}$ diameter, separated by silica spacers of $100 \mu \mathrm{m}$ thickness. The prepared sample was connected to the end of the coaxial line, and then melted in situ and annealed for $5 \mathrm{~min}$ at $383 \mathrm{~K}$. Isothermal frequency scans were carried out from $383 \mathrm{~K}$ down to $273 \mathrm{~K}$, in steps of $10 \mathrm{~K}$. A Quatro Cryosystem (Novocontrol Technologies $\mathrm{GmbH}$ ) was used to control the temperature with a stability of $\pm 0.2 \mathrm{~K}$ for both low and high frequency domains.

\subsection{Molecular Dynamics simulations (MD)}

Molecular dynamics (MD) simulations have been performed using the DL_POLY package ${ }^{36}$ and the GAFF force field (General Amber Force Field) ${ }^{37}$. All details about the MD simulations parameters and procedures employed are described in our previous work ${ }^{35}$ so we give here only the essential information. The modelled system possesses a total number of $\mathrm{N}=64$ molecules composed of S(-) Nac-MBA and $\mathrm{R}(+)$ Nac-MBA molecules in order to obtain different enantiomeric excesses. In the present work, MD simulations have been performed on systems composed of 32, 40, 48, 56 and 64 S(-) Nac-MBA molecules corresponding respectively to ee $=0 \%, 25 \%, 50 \%, 75 \%$ and $100 \%$. MD simulations were performed at five temperatures $500 \mathrm{~K}, 450 \mathrm{~K}, 400 \mathrm{~K}, 360 \mathrm{~K}$ and $340 \mathrm{~K}$. The 
equilibration/production times range from $10 / 200$ ns to $20 / 800$ ns from the highest to the lowest investigated temperature. The Nac-MBA densities in the liquid state determined in the present study are found identical for all ee and correspond to the values reported in our previous study ${ }^{35}$.

\section{Results and Discussions}

\subsection{Thermal and structural characterizations}

The calorimetric characterization of Nac-MBA was performed by DSC, MT-DSC and FSC. Here we detail some results obtained by DSC at different ee from ee $=0 \%$ up to ee $=100 \%$.

Figure 1 depicts the thermal behavior of both racemic mixture and pure enantiomer during heating at $10 \mathrm{~K} / \mathrm{min}$. Upon heating, the pure enantiomer and racemic mixture illustrate different thermal signatures. The single endothermal peak observed in the signal of ee $=100 \%$ is associated to the melting of pure enantiomer crystals. The melting event appears at $\operatorname{Tm}(\mathrm{R}$ or $\mathrm{S})=373 \mathrm{~K}$ with an enthalpy $\Delta \mathrm{H}_{\mathrm{m}}=(20.1 \pm 0.3)(\mathrm{kJ} / \mathrm{mol})$ and $\Delta \mathrm{S}_{\mathrm{m}}=0.054(\mathrm{~kJ} /(\mathrm{mol} . \mathrm{K}))$ the corresponding entropy. For the racemic mixture, three thermal phenomena appear-Druot et al. ${ }^{38}$ reported this complex behavior of the racemic composition upon heating but could not assigned for sure the nature of every phenomenon. So far, two possibilities appear for the first endotherm: either the reversible and stable three-phase equilibrium (eutectoid type) conglomerate-racemic compound: $\langle R\rangle+\langle S\rangle \leftrightarrow$ $<R S>$ or ii) the metastable melting of the conglomerate. The endothermal peak is directly followed by a low magnitude exothermal event suggesting crystallization or reorganization process. The last phenomenon that occurs at $348 \pm 2 \mathrm{~K}$ is associated to the melting of the racemic compound with a melting enthalpy $\Delta \mathrm{H}_{\mathrm{m}}=(16.3 \pm 0.8) \mathrm{kJ} / \mathrm{mol}\left(\Delta \mathrm{S}_{\mathrm{m}}=0.047 \mathrm{~kJ} /(\mathrm{mol} . \mathrm{K})\right)$. These results may suggest that there is a continuous transformation of the stable conglomerate towards the racemic compound, engaging at least two intermediate processes (melting of the residual conglomerate and recrystallization towards the racemic compound). This hypothesis is consistent with the fact that, due to the poor diffusion rate in solids, the eutectoid transition may not be complete, and the remaining proportion of $R$ and $S$-enantiomers melts as soon as the melting temperature of the conglomerate is achieved. 


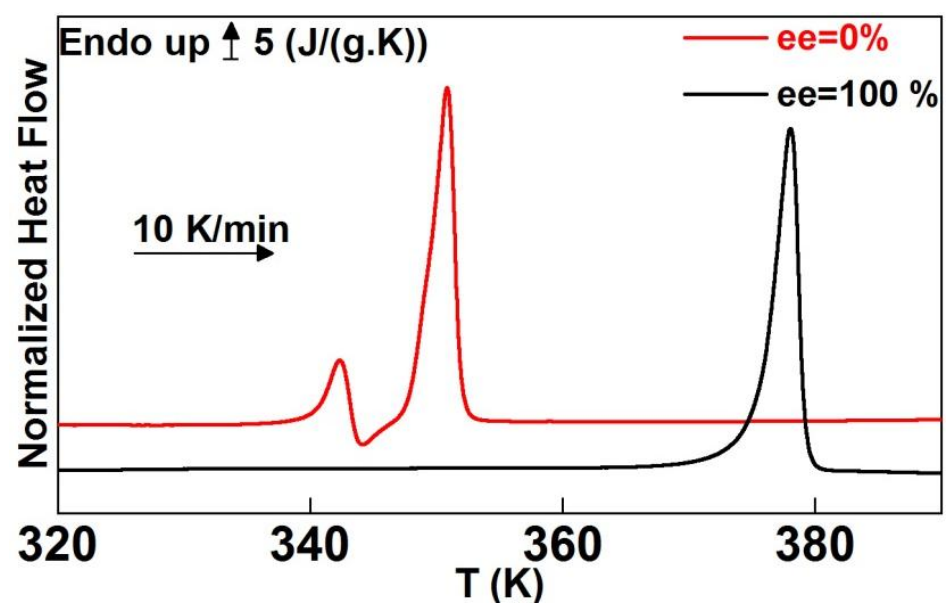

Figure 1: DSC Heating scans of pure enantiomer and racemic mixture at $10 \mathrm{~K} / \mathrm{min}$.

Indeed, during the heating of the stable conglomerate, there is a progressive transformation towards the racemic compound (but not completely). As soon as the melting temperature of the conglomerate is reached, all the residual $R$ and $S$-enantiomers that did not undergo the eutectoid transition melt and recrystallize as a racemic compound.

In order to evaluate the effect of high cooling rate (and hypothetically the effect of the sample size used for FSC) on the eutectoid invariant, succinct FSC measurements were performed from both crystals of the conglomerate and the racemic compound. Figure 2 illustrates the heating scan of the conglomerate and the racemic compound at $30000 \mathrm{~K} / \mathrm{min}$.

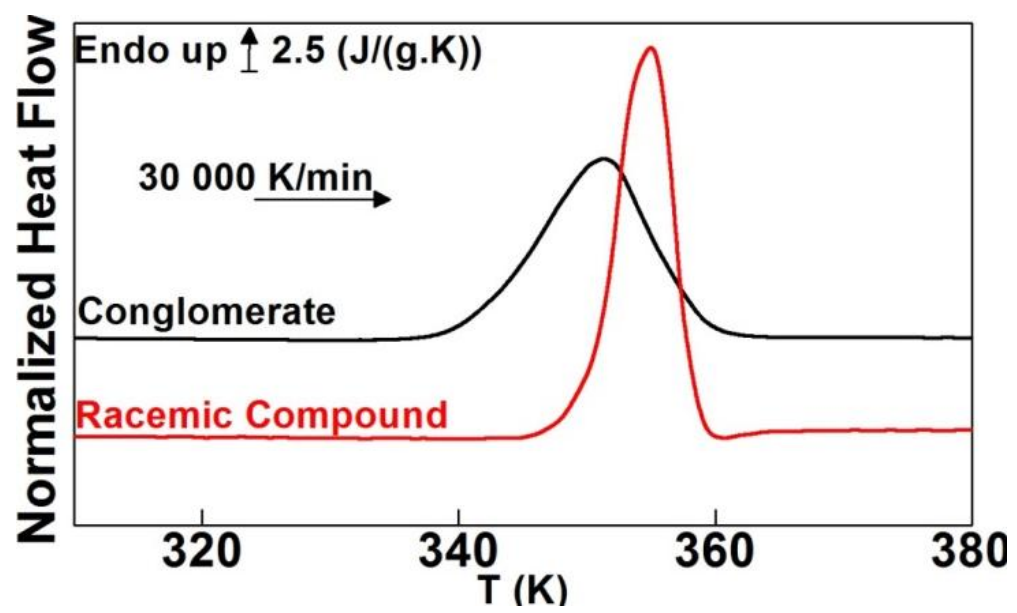

Figure 2: Heating scans at $30000 \mathrm{~K} / \mathrm{min}$ of both conglomerate and racemic compound crystals

The sample masses were estimated to be lower than $20 \mathrm{ng}$. The procedure of mass determination is well explicated in the work of Monnier and co-authors ${ }^{39}$. Upon heating, the conglomerate presented 
a single broad endothermal peak with an onset at $T_{m}=341 \mathrm{~K}$ associated to the melting of the conglomerate. The racemic compound presents a single endothermal peak at $\mathrm{T}_{\mathrm{m}}=350 \mathrm{~K}$ related to the melting. Consequently, no obvious transition from the conglomerate towards the racemic occurred. The thermal fluctuation imposed to the conglomerate is fast enough in such a way that the kinetic of the solid - solid transition cannot follow and only the metastable melting of the conglomerate can be evidenced. For the case of the racemic compound, only the expected melting peak is observed.

TR-XRD experiments were performed in order to follow the temperature evolution of the conglomerate towards the racemic compound (see figure 3). As temperature increases ( $>293 \mathrm{~K}$ ), there is a progressive appearance of characteristic peaks associated to the racemic compound and conversely some characteristic peaks related to the conglomerate disappeared in such a way that both phases may coexist before melting. Thus, the conglomerate evolves progressively towards the racemic compound as temperature increases. These results are in adequacy with those of Druot and coworkers ${ }^{38}$. Moreover, the co-existence of both phases (conglomerate and racemic compounds) from at least $50^{\circ} \mathrm{C}$ up to $70^{\circ} \mathrm{C}$ highlighted the difficulty to complete eutectoid transition due to the poor diffusion rate at the solid state (especially at large scale) and could explain the rather complex behavior upon heating characterized by DSC.

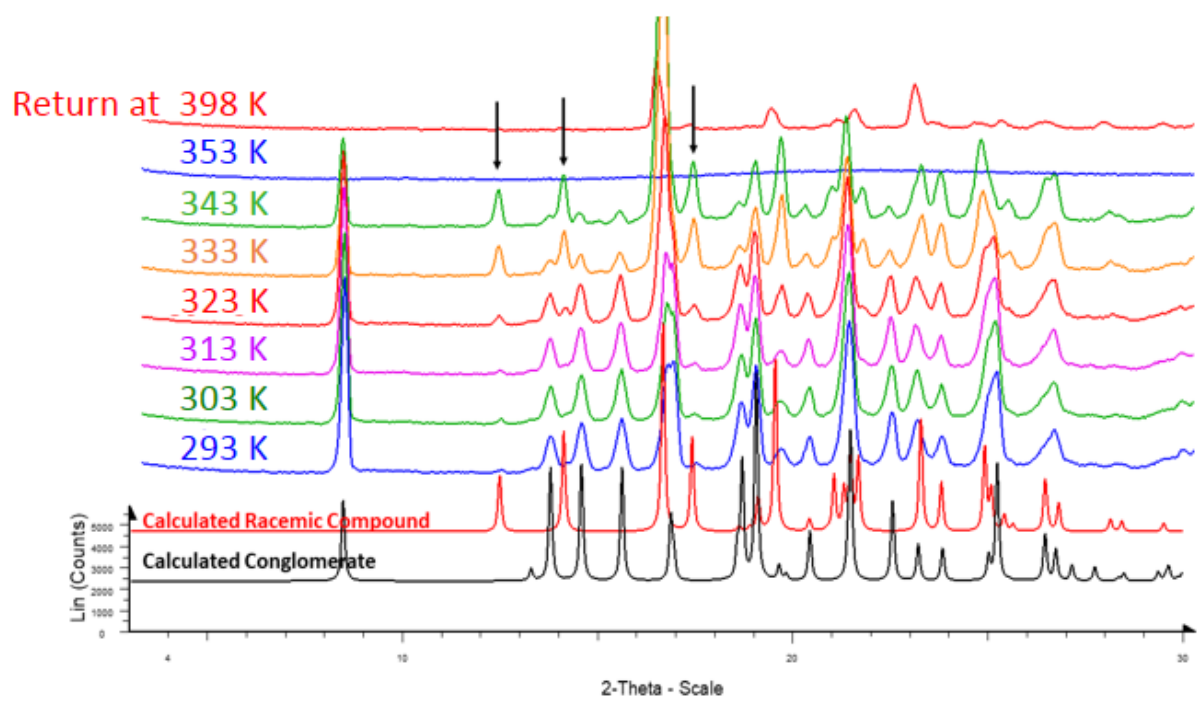

Figure 3: TR-XRD of the stable conglomerate showing a progressive evolution of the conglomerate towards the racemic compound upon heating. The black arrows point out the characteristic peaks of the racemic compound 


\subsection{Glass forming ability (GFA)}

Figure 4 illustrates the behavior upon melt cooling at $10 \mathrm{~K} / \mathrm{min}$ for various ee. While in the case of the racemic mixture, no crystallization occurred, samples at higher ee undergo partial or complete crystallization.

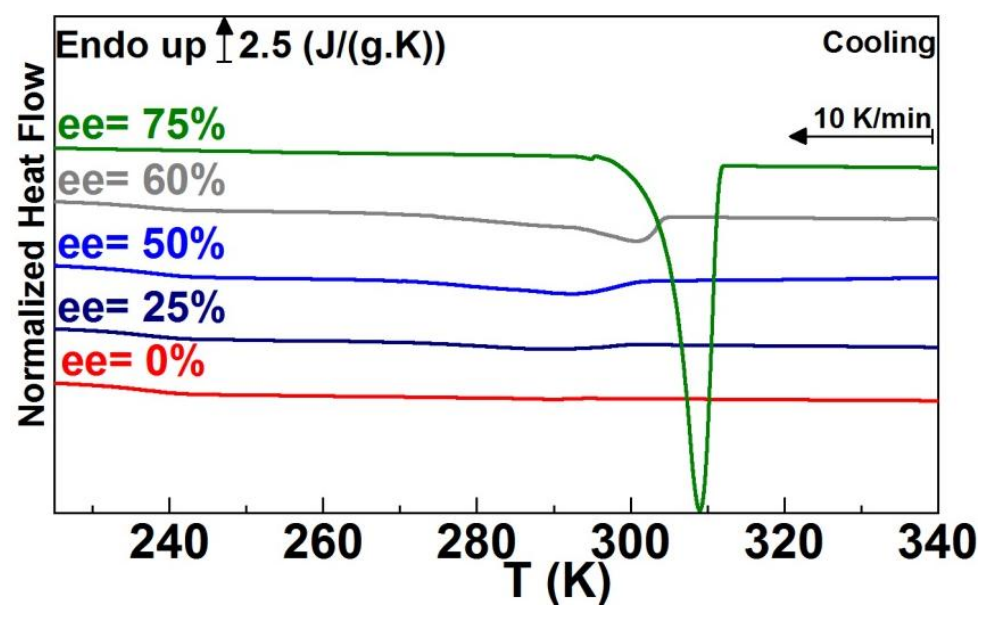

Figure 4: Thermal behavior upon cooling at $10 \mathrm{~K} / \mathrm{min}$ for several ee

Upon cooling, the crystallization enthalpy increases with the ee, and the crystallization temperature shifts towards lower temperatures as the ee decreases. These results suggest that, higher cooling rate than $10 \mathrm{~K} / \mathrm{min}$ are required in order to vitrify the samples with an ee $>0 \%$, and the cooling rate needed to inhibit crystallization increases proportionally to the ee. As illustrated in figure 5 the pure enantiomer undergoes crystallization even at $200 \mathrm{~K} / \mathrm{min}$, while vitrification of racemic Nac-MBA can easily be reachable at cooling rate $\geq 5 \mathrm{~K} / \mathrm{min}$ (With a $\mathrm{Tg}=241 \mathrm{~K}$ )

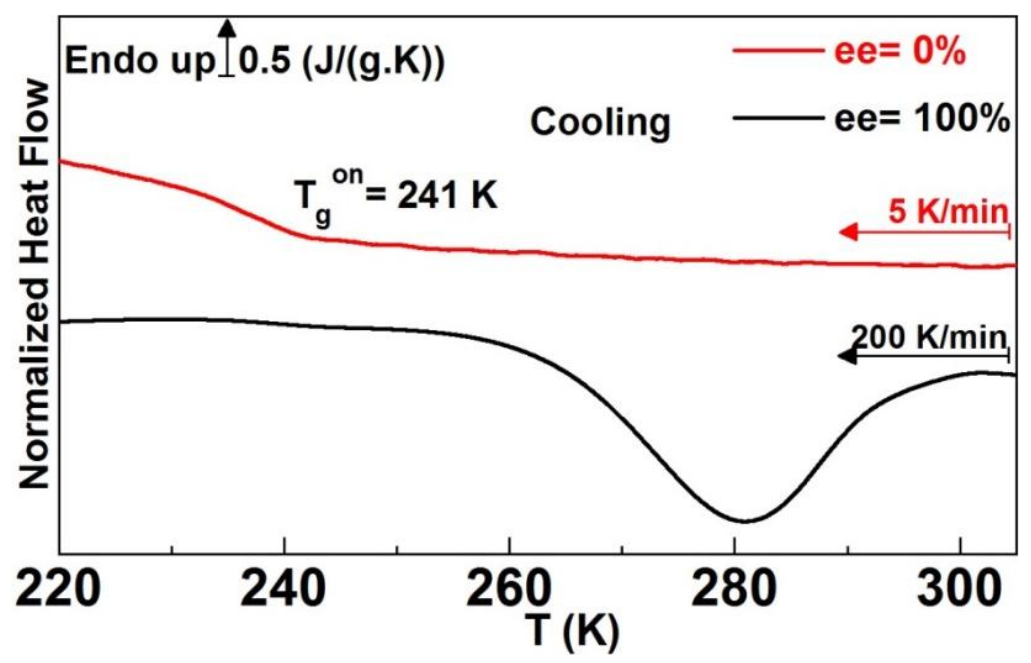

Figure 5: Thermal behavior upon cooling of both pure enantiomer and racemic mixture by classical DSC 
Such differences in the GFA between the pure enantiomer and the racemic composition has already been mentioned by some of us in a previous work for the case of another conglomerate forming system: 5 -ethyl-5-methylhydantoïn ${ }^{30}$. To our knowledge this is the second compound for which such behavior is revealed. The common feature between both systems is that they crystalize as stable conglomerates at room temperature. The main explanation of this behavior remains kinetic. In fact, crystallization occurs only when the molecules of the liquid phase (disorder) reorganize in a certain way that they can build the long-range order of the crystal. This reorganization seems disturbed and delayed by the presence of the counter enantiomer. In the case of a pure enantiomer, all neighbor molecules are of the same chirality, therefore, crystallization may occur more rapidly than in a mixture of opposite enantiomers. Therefore, one can consider that the GFA is function of the enantiomeric composition and increases with decreasing ee. In this case, racemic Nac-MBA can be considered as a good glass former when the pure enantiomer is referenced as a poor glass former. These results are typically different from the behavior expressed in other chiral systems crystallizing as either as racemic compounds or solid solutions. In the case of Limonene (crystallizing as a metastable solid solution), Galis and coworkers ${ }^{32}$ deported that the crystallization behavior upon cooling is very random and independent of the enantiomer ratio. The study performed by Viel et al.

${ }^{33}$ highlighted the fact that the crystallization tendency is well pronounce at the racemic composition than for the pure enantiomers in the case of diprophylline that crystallize as a racemic compound. It seems that for conglomerate forming systems, the crystallization tendency of the pure enantiomers is mostly favored for kinetic reasons in comparison with a racemic composition.

\subsection{Recrystallization from the amorphous state}

In order to vitrify the liquid, appropriate cooling rate was applied regarding the enantiomeric composition. For ee $<80 \%$ a cooling rate of $100 \mathrm{~K} / \mathrm{min}$ was applied, and for ee $>80 \%$ cooling rate higher than $250 \mathrm{~K} / \mathrm{min}$ have to be considered to inhibit the melt crystallization. After the cooling step, the amorphous glass was then heated at $10 \mathrm{~K} / \mathrm{min}$. Figure 6 presents the thermal signal upon heating at $10 \mathrm{~K} / \mathrm{min}$ of the amorphous sample at different ee. Upon heating, at least three thermal events were evidenced. The glass transition appears at $\mathrm{Tg}^{\mathrm{mid}}=240 \pm 1 \mathrm{~K}$ independently of the enantiomeric composition. Cold crystallization occurs at higher temperature depending on the ee; the crystallization temperature is shifted towards higher temperature with the decrease of the ee. The exothermal crystallization peak is followed by an endothermal event associated to the metastable eutectic invariant $T_{E}=347 \pm 2 \mathrm{~K}$ (the onset temperature is independent of the ee) of the racemic compound. The last endothermic peak is associated to the liquidus phenomenon (the peak temperature is considered as the liquidus temperature). 


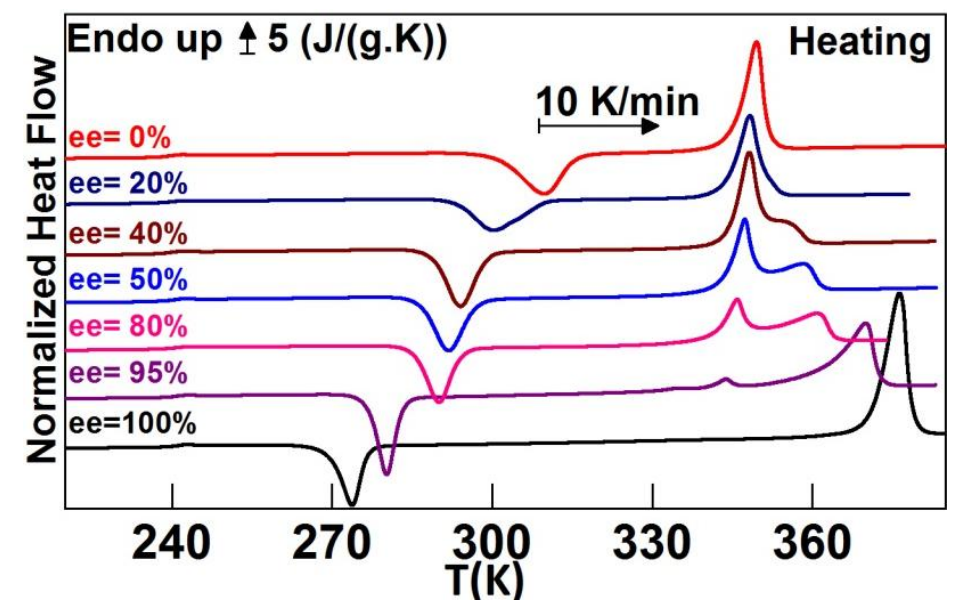

Figure 6: Heating at $10 \mathrm{~K} / \mathrm{min}$ from the amorphous state of the samples at several ee. The meltcooling was performed at $100 \mathrm{~K} / \mathrm{min}$ for ee $<80 \%$ and $250 \mathrm{~K} / \mathrm{min}$ for ee $>80 \%$

For every composition, recrystallization from the amorphous state did not lead to the eutectoid invariant, only the eutectic invariant associated to the melting of the racemic compound and residual pure enantiomer was evidenced. Thus, the recrystallization from the amorphous state leaded to the appearance of the racemic compound in its metastable domain. These hypotheses are consistent with the TR-XRD diffractogram measurement performed during heating of the glassy material. Racemic Nac-MBA was melted and quenched in liquid nitrogen. The diffractogram was registered from $253 \mathrm{~K}$ up to $353 \mathrm{~K}$ by step of $10 \mathrm{~K}$, see Figure 7.

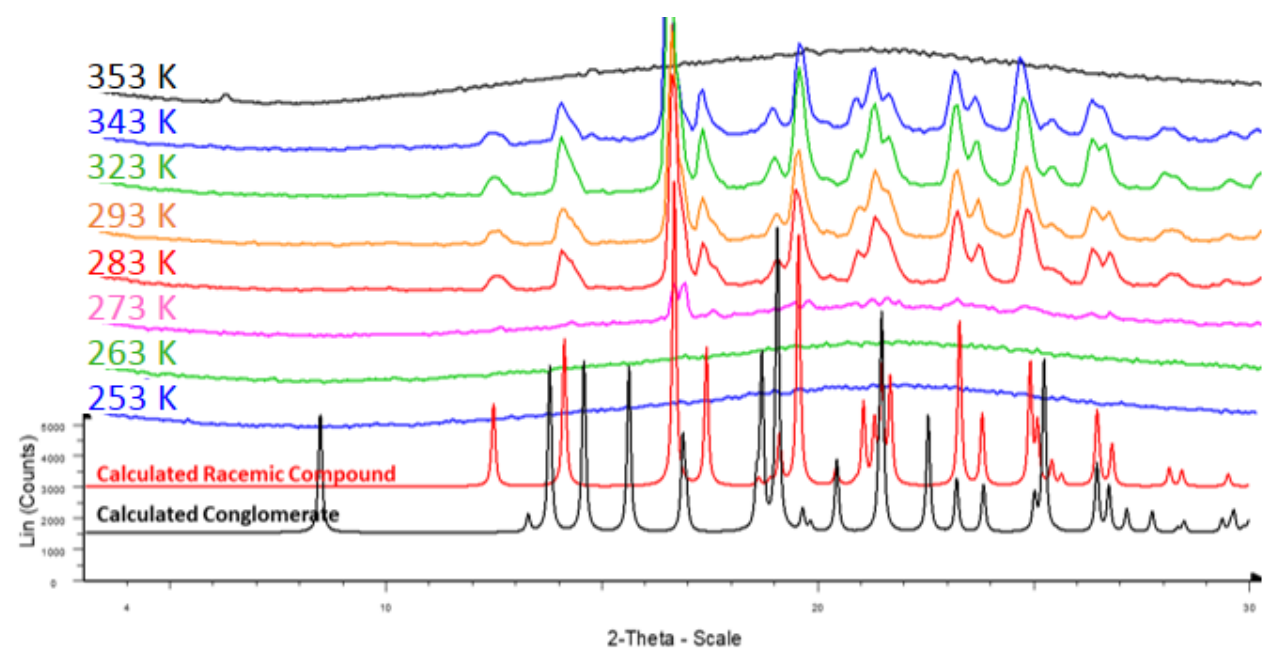

Figure 7: TR-XRD of the amorphous sample (ee=0\%) recrystallizing as a racemic compound upon heating

At $253 \mathrm{~K}$ and $263 \mathrm{~K}$, only the amorphous halo was evidenced, suggesting that vitrification was successfully achieved upon quenching. The initial amorphous sample recrystallizes between $273 \mathrm{~K}$ and $283 \mathrm{~K}$ towards the racemic compound. No appearances of characteristic peaks corresponding to 
the conglomerate were evidenced. Thus, racemic amorphous Nac-MBA recrystallizes as a racemic compound. All thermal events are represented in a-phase-diagram-like plot in figure 8 as follow:

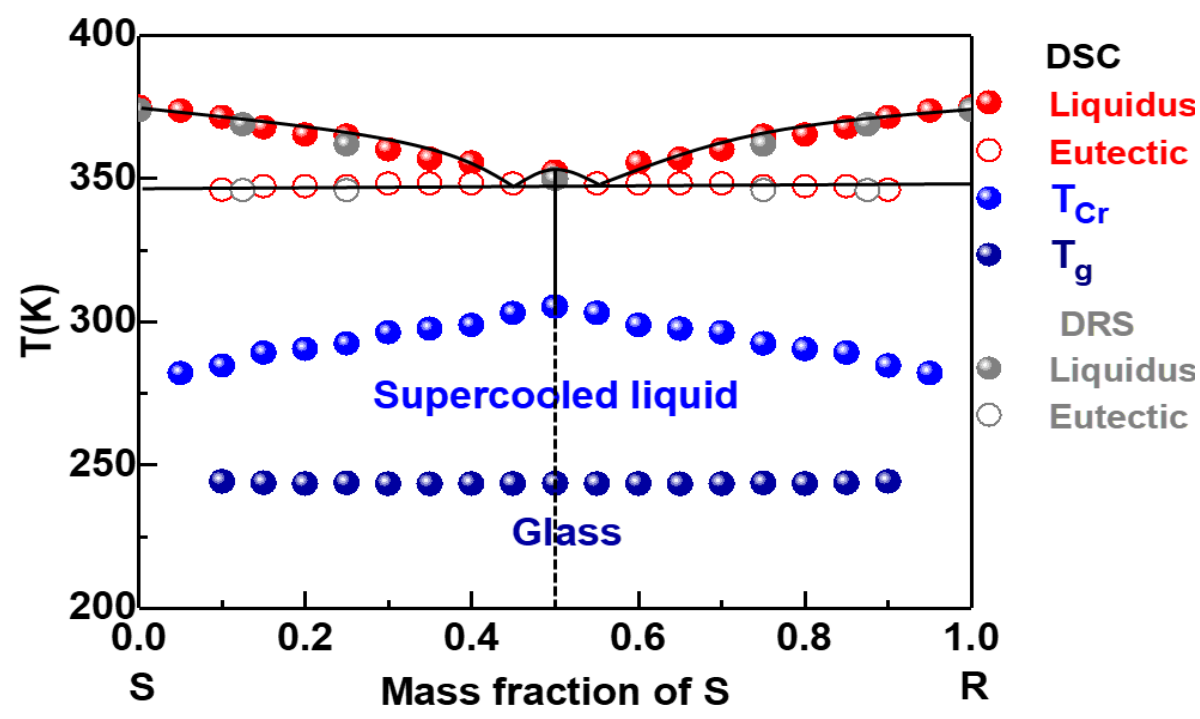

Figure 8: Metastable binary phase diagram between the two chiral antipodes, established from the heating of the amorphous glass. $T_{g}$ and $T_{c r}$ are respectively the glass transition and crystallization temperatures obtained by calorimetric investigations

Regardless the cooling rate, the melted sample always recrystallized as a racemic compound. This observation seems very contradictory regarding the weakly crystallogenic tendency of the racemic mixture since it hardly crystallizes upon cooling. Even though the counter enantiomer acts like a configurational impurity during the melt-cooling, the possibility of forming centrosymmetric pairs (racemic compound) is highly favored when the system is in convenient crystallization conditions. However, this behavior seems accurate with the high occurrence of racemic compounds $(95 \%$ of occurrence) compared to conglomerate systems (5\%) ${ }^{7,12}$. The preponderance of racemic packing over homochiral structure is still an unsolved dilemma in the field of stereochemistry. Based on a set of 8 pairs of density values, Wallach stated that racemic packing are more stable than their homochiral counterpart since they are much denser ${ }^{40}$. Unfortunately, Nac-MBA system does not follow the Wallach's rule since the pure enantiomer is as denser as the racemic compound (with less than $1 \%$ difference). Statistically, only $28 \%$ of the available space groups are compatible with an homochiral arrangement since chirality do not authorize any explicit or implicit inversion operation of symmetry ${ }^{7}$. Contrary, racemic compounds can fit without any restriction in all the 230 existing space groups. Without any consideration of the molecular handedness Kitaigorodskii demonstrates the fact that most of existing molecular compounds preferentially crystallize in space groups with symmetry elements as translation, inversion center, glide mirrors and direct axes. Typically, the space groups $P 2_{1} / c$ and $P \overline{1}$ count for more than $50 \%$ occurrence of all known molecular organic crystal 
structure. Regarding these statistical considerations, the occurrence of racemic compounds seems highly preponderant. Another issue regarding the crystallization behavior of Nac-MBA we wish to highlight concerns the kinetic aspect. This seems at our point of view the most important issue governing the crystallization behavior of racemic Nac-MBA as a metastable racemic compound. In fact, the racemic melt is composed of both enantiomers in equal proportions. For the racemic melt to crystallize as a homochiral packing the R enantiomer must be surrounded by the "suitable" enantiomer same for the $S$ enantiomer. Half of the time this condition is mostly not fulfilled. The non-suitable enantiomer has to be changed by a suitable enantiomer in order to pursue crystallization. The presence of this "non-suitable" neighbor enantiomer will therefore inhibit -at least retard- the formation of viable nuclei and their growths. By contrast, for a racemic compound, the probability for an enantiomer to be in the direct environment of a suitable-enantiomer is much higher. The enantiomer does not need to be changed by another when it is not in the suitable site but just needs to "switch and slide" in the good crystallographic site. As the crystallization of homochiral crystals is kinetically unfavorable, the liquid will evolve towards the easiest and immediate available metastable equilibrium state in accordance with the Oswald's law of stages.

\subsection{Phase transitions characterized by DRS}

The recrystallization and melting phenomena at several ee were probed by DRS. Measurements were performed during melt cooling/heating cycles from $388 \mathrm{~K}$ down to $173 \mathrm{~K}$ at $1 \mathrm{~K} / \mathrm{min}$ for various ee. Figure 9 depicts the temperature evolution of the real permittivity $\varepsilon^{\prime}$ at $117 \mathrm{kHz}$ upon cooling and heating scan. Upon melt cooling, $\varepsilon^{\prime}$ slightly increases with decreasing temperature in the supercooled-liquid region but suddenly begins to drop for more than one order of magnitude at an ee dependent temperature. The decrease of $\varepsilon^{\prime}$ appears very abrupt for high enantiomeric ratio (ee = $100 \%$ and ee $=75 \%$ ) but progressively for ee $\leq 50 \%$. These behaviors suggest a decrease in degrees of freedom associated to recrystallization of the supercooled-liquid. Moreover, the crystallization temperature increases as the ee decreases. As already mentioned from DSC cooling scans, high ee (ee $>50 \%$ ) possess strong crystallization tendency or poor GFA compared to lower ee (ee $\leq 50 \%$ ).

The heating behavior of the recrystallized samples revealed different thermal phenomena. In the case of the pure enantiomer, $\varepsilon^{\prime}$ strongly increases as soon as the melting point was reached. For lower ee, the increase of $\varepsilon^{\prime}$ is independent of the composition. Such behavior is associated the eutectic invariant equilibrium. Furthermore, the onset temperature of $\varepsilon^{\prime}$ increase is in good agreement with the eutectic temperature measured from DSC data. When complete melting is achieved, $\varepsilon^{\prime}$ slightly decreases with increasing temperature. The onset temperature at which $\varepsilon^{\prime}$ starts 
to decrease smoothly is here mentioned as the liquidus temperature. All these thermal events are in very good agreement with the DSC data were reported figure 8.
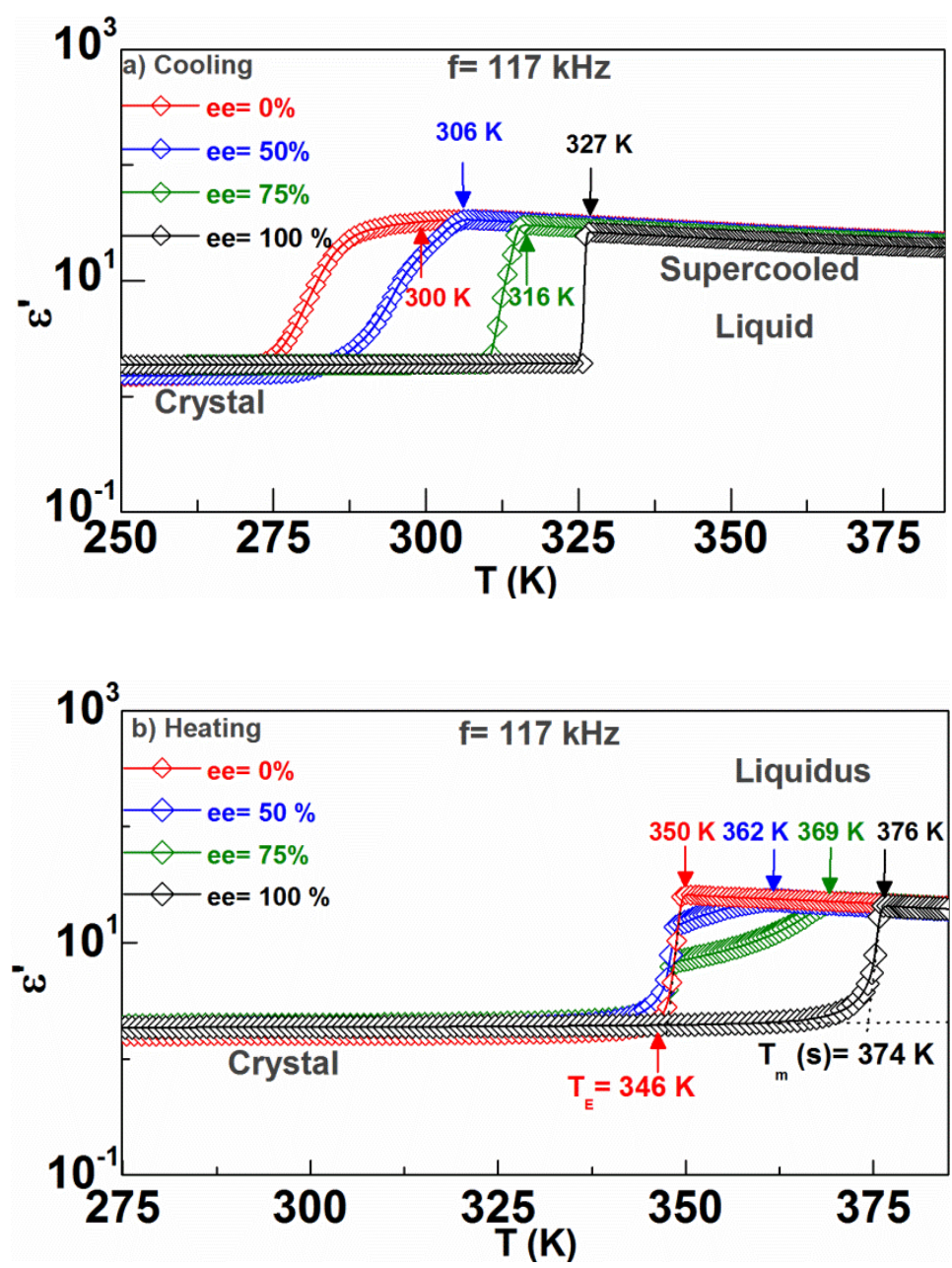

Figure 9: Temperature evolution of the real dielectric permittivity $\varepsilon^{\prime}$ upon a) cooling and b) heating cycles at $1 \mathrm{~K} / \mathrm{min}$ for a frequency $\mathrm{f}=117 \mathrm{kHz}$. The arrows mark the crystallization temperature and the eutectic or liquidus temperatures for the cooling and heating data respectively. $T_{E}$ and $T_{m}(S)$

represent the eutectic temperature and the melting temperature of the pure enantiomer respectively 


\subsection{Molecular Mobility Characterization by DRS}

The molecular mobility of Nac-MBA system was partly investigated in our previous work ${ }^{35}$ over a wide temperature (from the glassy to the liquid state) and frequency range for an ee $=50 \%$. In the glassy state, two secondary processes were evidenced: the fast $\gamma$ relaxation and slow $\beta_{J G}$ associated respectively to the fluctuation of the acetyl group and the reorientation of the molecule as a whole. The structural $\alpha$ process and the Debye (D) relation were evidenced in the supercooled and liquid states. The $\alpha$ relaxation is associated to the dynamic of the glass transition and the Debye D process is associated to the transient formation/reformation of linear $\mathrm{H}$-bonded molecules.

In the present paper, we propose to analyze the molecular mobility of the model compound at several enantiomeric excesses. This can help to shed light on the impact of chirality (if measurable) on the molecular mobility of Nac-MBA by evaluating the relaxation time of the various processes expressed at variable ee. More details about the analysis of the relaxation processes and their temperature dependencies are displayed in the supplementary information.

The relaxation time temperature dependence of the processes as a function of the enantiomeric composition is plotted in the relaxation map illustrated in figure 10. For the sake of clarity and simplicity the relaxation map is displayed into two figures reporting the temperature dependency of a) the secondary processes relaxation time, $b$ ) the $D$ and $\alpha$ relaxation times respectively.

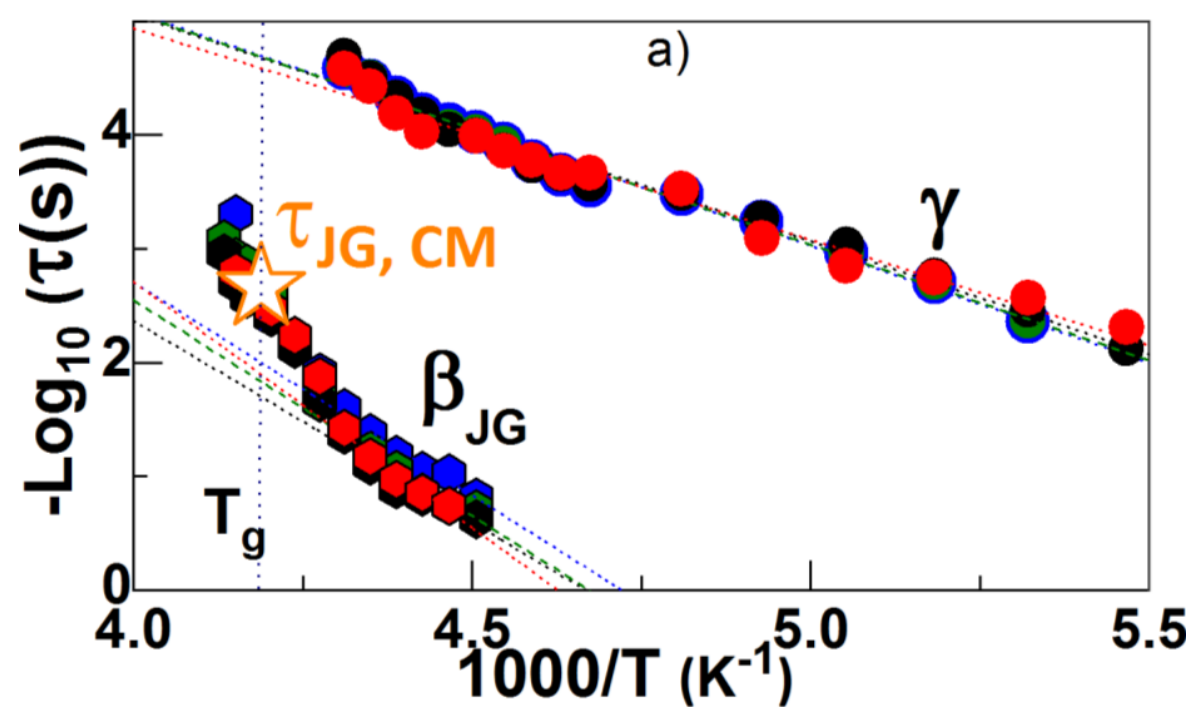




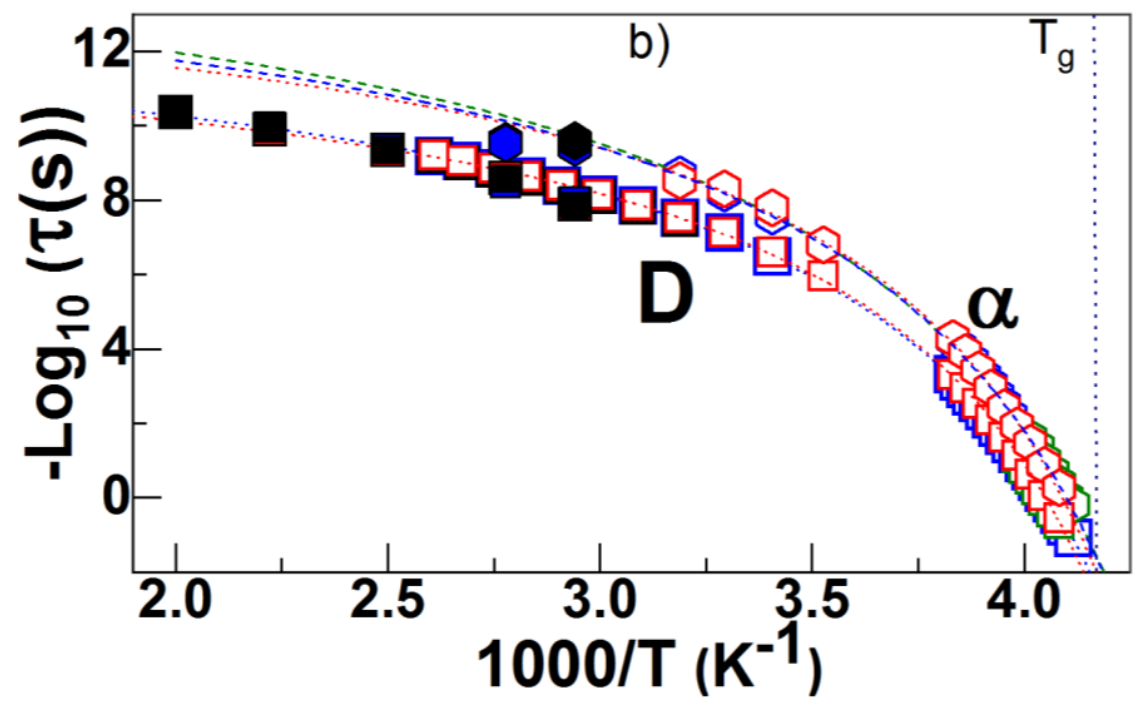

Figure 10: Temperature dependency of the relaxation time of a) secondary $\beta_{J G}$ and $\gamma$ processes $b$ ) Debye and $\alpha$ processes at different ee from DRS and MD simulations (filled symbols in figure b). Each color is attributed to an ee, black for $e e=100 \%$, green for $e e=75 \%$, blue for $e e=50 \%$ and red for ee $=0 \%$. The orange star represents the JG relaxation time calculated at $T_{g}$ from the Coupling Model ${ }^{41}$.The dot lines give the fit of the Arrhenius and VFTH equations to the corresponding data (same color as the data)

Regarding the information given by the relaxation map, the following picture can be drawn: the relaxation time and their temperature dependency almost superimposed (within the limit of uncertainties) at all the analyzed ee, independently of the relaxation process. This may suggest that molecular mobility is not affected by the enantiomeric ratio whatever the microscopic origin of the relaxation process; intra or intermolecular localized motions for secondary processes and cooperative or transient intermolecular motions expressed in the supercooled-liquid state.

Thus, the relaxational landscape of Nac-MBA is insensitive to chirality. Such result has already been reported for different chiral systems crystallizing as stable racemic compounds such as ibuprofen ${ }^{28}$ and diprophyline ${ }^{27}$. One may ask if the situation of Nac-MBA could be different if the system recrystallized as a homochiral packing. In other words, does chiral discrimination in the solid state still persist in the amorphous state and if yes, to what extent? To answer this question, chirality impact has to be investigated from a model stable conglomerate that do not undergoes any metastable phase transformation or a racemic compound that becomes a metastable conglomerate upon recrystallization. This is not an easy task considering the poor occurrence of conglomerate forming systems. If for a hypothetical system presenting the described behavior, any difference 
appears in the relaxation time, then, chiral discrimination may also be expressed in the amorphous state.

\subsection{MD simulations}

\section{Static and dynamical dipolar properties}

Static and dynamical dipolar properties of the different enantiomeric mixtures in the liquid state have been investigated based on the behavior of the Nac-MBA dipole moments and similarly to reference ${ }^{35}$. In particular, we have calculated the average dipole moment $\langle\mu\rangle$ of Nac-MBA molecules, the dipole-dipole correlations using the Kirkwood correlation factor $\mathrm{G}_{\mathrm{K}}$ and the time-dependent properties using the dipolar correlation function $\langle\vec{M}(t) \cdot \vec{M}(0)\rangle$ where $\vec{M}(t)$ is the total dipole moment of the system ${ }^{42}$. This function allows probing the collective dynamics of Nac-MBA dipoles ${ }^{35}$. On a first approximation, $\langle\vec{M}(t) \cdot \vec{M}(0)\rangle$ can be compared to the Fourier transform of the complex frequency dependent permittivity measured experimentally by DRS ${ }^{42}$. Within the limit of uncertainties, all data (average dipole moment $\langle\mu\rangle$, Kirkwood correlation factor $\mathrm{G}_{\mathrm{K}}$ and dipolar timedependent correlation functions $\langle\vec{M}(t) \cdot \vec{M}(0)\rangle)$ superimposed for all ee and all temperatures (data not shown). All data are similar to those reported in ${ }^{35}$ for ee $=50 \%$. From MD computations, no impact of the enantiomeric excess is thus detected on the different static and dynamical dipolar properties.

These numerical results are well in line with the DRS data obtained in the present study which also reveal that dipolar properties of Nac-MBA are not impacted by chirality. The few dielectric relaxation spectroscopy investigations which have been performed on the impact of chirality have generally reported similar features between homochiral and racemic systems in the liquid, undercooled liquid or glassy state of many different molecular systems ${ }^{27,29,43-47}$. The glass transition temperature particularly remains quite constant ${ }^{7,27,29,30,32}$ whatever the enantiomeric excess for many different systems as found in the case of Nac-MBA.

It strongly contrasts with the vitrification/crystallization tendencies of supercooled pure enantiomeric and racemic mixtures which are often entirely different ${ }^{27,29-31}$. However, it should be noted that some differences between homochiral and racemic systems have been shown from DRS. In the specific case of ibuprofen ${ }^{28}$, most of the dynamical dipolar properties are found to be nearly identical in the homochiral and racemic systems. But the static dipolar properties and the intensity of the Debye-like process in particular are clearly found to be more intense in the racemic mixture than in the enantiomerically pure ibuprofen. For this system, based on MD simulations, the difference seems to originate from the presence of the syn and anti-conformations of the $-\mathrm{COOH}$ moiety of the ibuprofen molecule and its link to the peculiar Debye-like dynamical process detected in this 
compound. The difference is explained by the higher population of the anti-conformation (with the higher dipole moment) and the lower population of hydrogen bonded cyclic dimers that can be transiently formed in the racemic mixture. Some experimental evidences of reliable difference between homochiral and racemic systems from DRS have been also reported in a few other systems such as 3 -methylcyclohexanone ${ }^{44}$, menthone ${ }^{44}$, tartrate ${ }^{45}$, and ketoprofen ${ }^{26}$. The possible impact of chirality on dipolar properties thus still appears an open question and seems possibly dependant of the chemical specificities of the molecules.

\section{Hydrogen bonding structures}

Nac-MBA molecules possess an amide group from which they may form intermolecular $\mathrm{HBs}^{35}$. From MD computations an arbitrary criterion is required in order to define if an HB is formed or not. In the present study, two Nac-MBA molecules are considered to be H-bonded if: (i) the nitrogen-oxygen distance is less than $3.4 \AA$ (ii) the $(\mathrm{O} \cdots \mathrm{H}-\mathrm{N})$ angle is larger than $150 \mathrm{deg}$. These criteria are classically used in MD simulations and take into account distorted and weak HBs in statistics ${ }^{48}$. Based on these criteria, HB statistics have been computed in order to determine the population of the different HB associating structures existing in the liquid state. Since the Nac-MBA molecule has a single- $\mathrm{NH}$ and a single $\mathrm{C}=\mathrm{O}$ group, the structure are mainly linear $\mathrm{HB}$ chains ${ }^{35}$ formed by a sequence of the $\mathrm{C}=0 . \ldots \mathrm{H}-\mathrm{N}$ motif. The fraction of HB clusters composed of $n$ Nac-MBA molecules has been computed for all investigated ee and temperatures and it is shown in Figure 11 at $\mathrm{T}=340 \mathrm{~K}$.

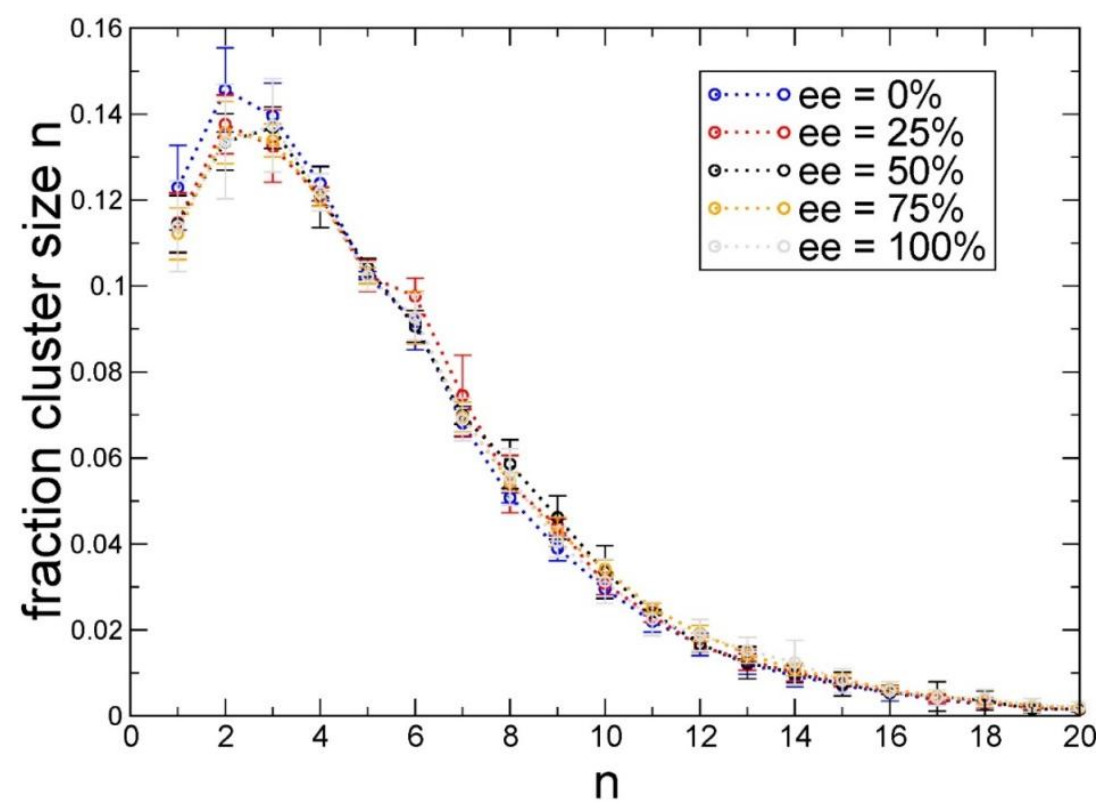

Figure 11: Fraction of HB clusters made of $n$ Nac-MBA molecules as determined from MD simulations for ee $=0 \%, 25 \%, 50 \%, 75 \%$ and $100 \%$ in the liquid state at $T=340 \mathrm{~K}$. A HB cluster of size $n$ is defined as an assembly of $n$ hydrogen-bonded Nac-MBA molecules. $n=1$ corresponds to an isolated molecule 
Within the limit of uncertainties, Figure 11 clearly reveals no impact of the ee on the overall population of HB clusters similarly to the static and dynamical dipolar properties (see above). In our previous work ${ }^{35}$, a detailed study has been already performed for ee $=50 \%$ about the population of HB clusters so we give here only the essential information. Overall, Figure 11 particularly shows that a large variety of HB clusters are detected ranging from isolated molecule $(n=1)$ to very large clusters made of $n=20$ molecules. Large clusters made of $n>10$ molecules have typically a short life $\mathrm{e}^{18,28}$. Their fraction remains really small at all investigated temperatures. At $T=340 \mathrm{~K}$, the most probable HB clusters are composed of only a few molecules $(n=2,3$ or 4$)$. The fraction of isolated molecules $(n=1)$ is significantly high at this temperature and progressively increases upon increasing temperature while the fraction of clusters of larger size decreases as reported in ${ }^{35}$. This trend is expected due to the increase of thermal agitation which tends to disrupt the hydrogen bonds. The opposite trend is also expected upon cooling below $340 \mathrm{~K}$ and the propensity of larger HB clusters should increase.

A detailed study of the fraction of homochiral and heterochiral HB dimers has been performed in the liquid state at $\mathrm{T}=340 \mathrm{~K}$ for ee $=0 \%, 25 \%, 50 \%$ and $75 \%$. It is recalled that one heterochiral dimer is composed of one S(-) Nac-MBA molecule hydrogen-bonded to one $\mathrm{R}(+)$ Nac-MBA molecule while one homochiral dimer is composed of either two S(-) Nac-MBA or two $\mathrm{R}(+)$ Nac-MBA HB molecules. The results are summarized in Figure 12 which shows the fraction of heterochiral HB dimers as function of the ee compared to the expected theoretical value assuming ideal equiprobability between all types of possible dimers (R-R, S-S and R-S). For example, assuming ee $=0 \%$ (same number of $R$ and $S$ Nac-MBA molecules) and ideal equiprobability between all types of possible dimers (R-R, S-S and RS), the expected fraction of dimers should be $25 \%, 25 \%$ and $50 \%$ for R-R, S-S and R-S dimers respectively. 


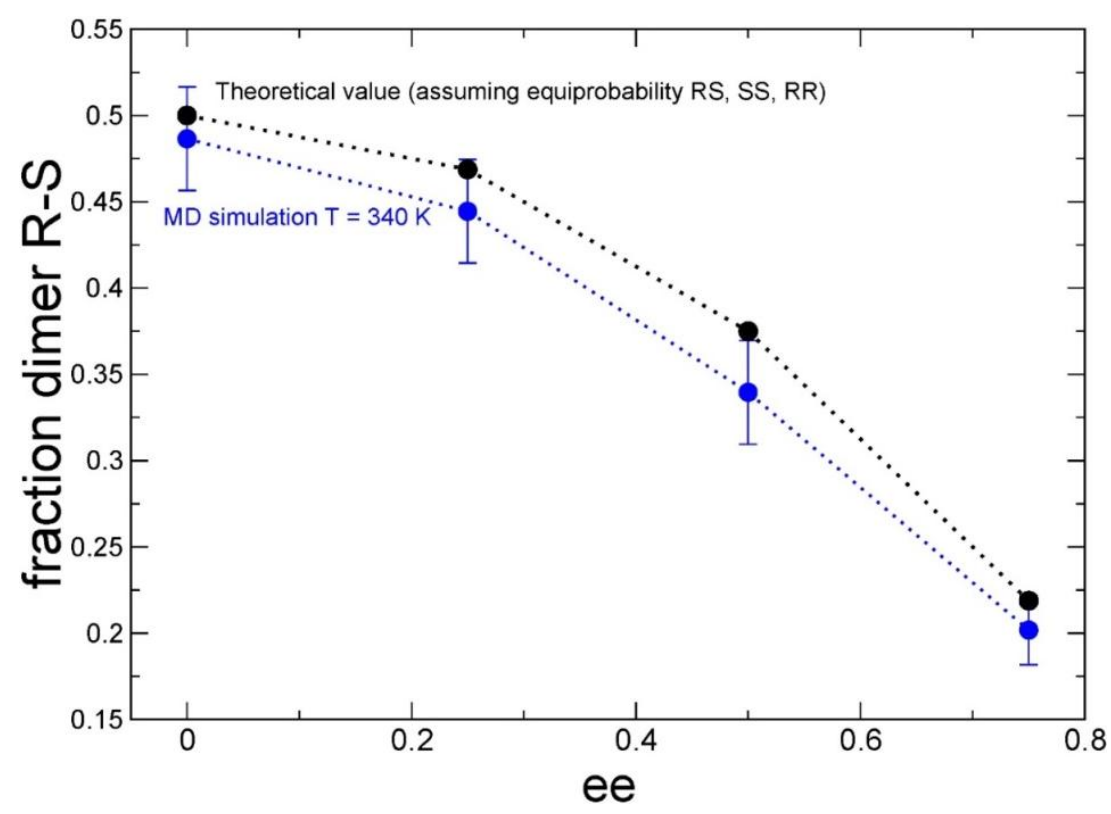

Figure 12: Fraction of heterochiral dimers formed in the liquid state at $T=340 \mathrm{~K}$ as determined from MD simulations for $e e=0 \%, 25 \%, 50 \%$ and $75 \%$. One heterochiral dimer is composed of one $S(-)$ Nac-MBA molecule hydrogen-bonded to one $R(+)$ Nac-MBA molecule. The expected theoretical value of the fraction of heterochiral dimers assuming ideal equiprobability between all types of possible dimers (R-R, S-S and R-S) is also shown for comparison

Interestingly, Figure 12 reveals that the fraction of RS dimers is systematically below the expected theoretical value. This systematic trend is confirmed at all investigation temperatures till at a temperature as high as $500 \mathrm{~K}$ at which the fraction still remains slightly below the expected theoretical (data not shown). It suggests that Nac-MBA homorochiral dimers (R-R or S-S) are slightly favored when compared to heterochiral dimers (R-S) which seems very consistent with the intrinsic nature of Nac-MBA to form a conglomerate but opposite to the crystallization trend from the amorphous state which leads to the appearance of the racemic compound in its metastable domain. It should be noted that the problem of the stability between heterochiral vs homochiral dimers has attracted a lot of consideration from DFT computations in the framework of the chiral discrimination through hydrogen-bonded ${ }^{49-51}$.

However, literature on simulations devoted to this subject does not clearly presents a uniform picture since the reported differences in energy between homochiral and heterochiral are often of the order of a few $\mathrm{kcal} / \mathrm{mol}$ and seems dependent on many factor including the molecular systems considered and its HB capabilities, the solvation effects, the computations details and the level of theory.

In order to study the impact of chirality on larger HB chains composed of $n=3,4$ or 5 molecules, we have searched from specific homochiral or heterochiral sequences in these structures. 
Sequences such as R-R-R (S-S-S) or R-S-R (S-R-S), R-R-R-R (S-S-S-S) or R-S-R-S (S-R-S-R) and R-R-R-R-R (S-S-S-S-S) or R-S-R-S-R (S-R-S-R-S) have been particularly searched. Results are shown in Figure 13 for ee $=0 \%$ at the different investigated temperatures.
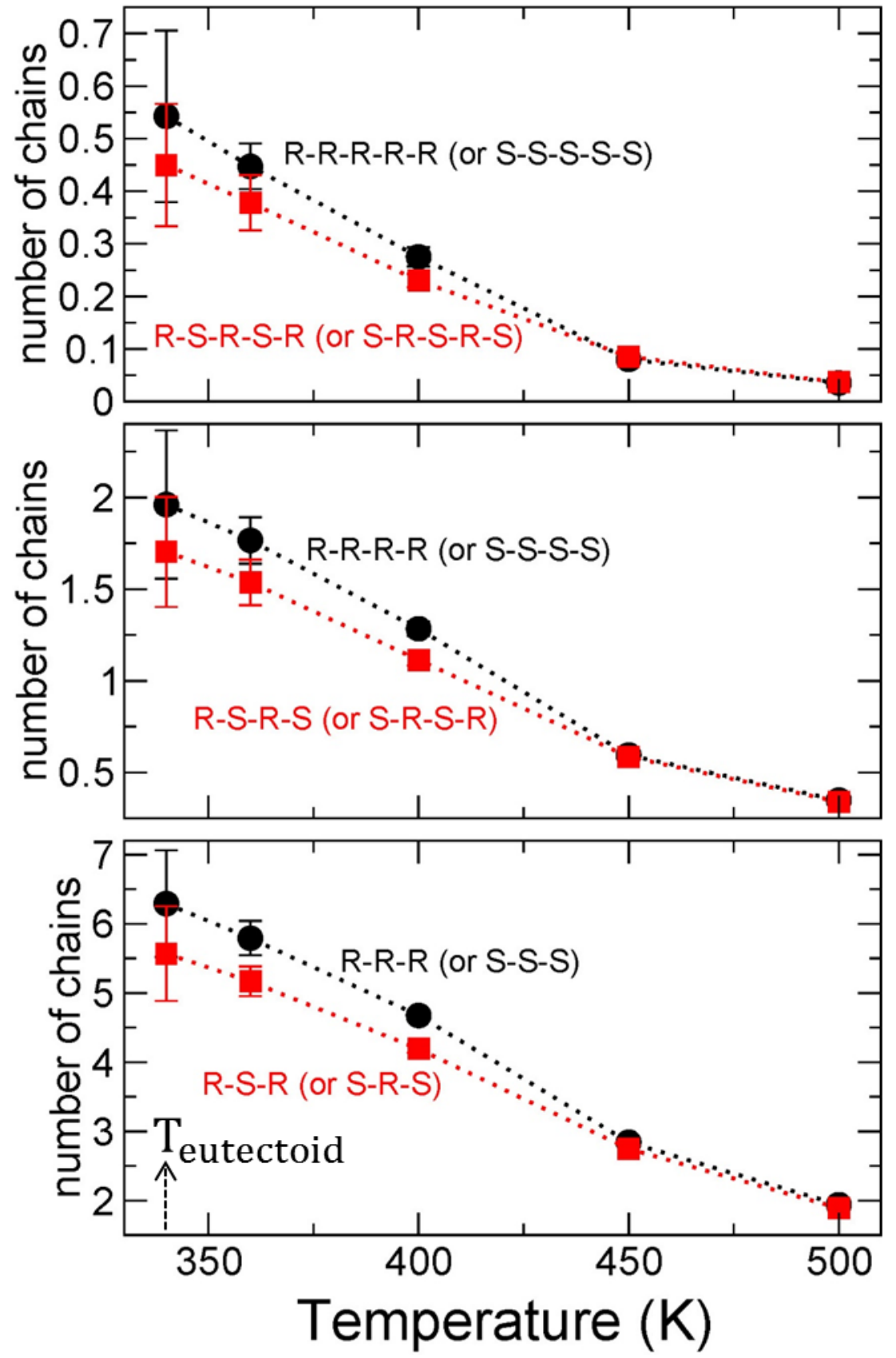

Figure 13: Number of homochiral or heterochiral HB chains formed in the liquid state at different temperatures as determined from MD simulations for ee $=0 \%$. A chain is defined as a sequence of hydrogen-bonded S(-) Nac-MBA or R(+) Nac-MBA molecules. For example, R-S-R represents a sequence of three hydrogen-bonded Nac-MBA molecules: $R(+)$ Nac-MBA, S(-) Nac-MBA and $R(+)$ Nac-MBA in that order 
This figure clearly demonstrates that the number of homochiral or heterochiral sequences slightly differs. The number of homochiral sequences (isotatctic $\mathrm{Hb}$ chains) is higher than the number of heterochiral sequences (syndiotactic and atactic $\mathrm{Hb}$ chains) especially at the lowest temperatures consistently with findings obtained for the dimeric associations. Interestingly, the separation between both types of sequences seems to increase upon decreasing temperature well in line with the property of Nac-MBA to form a stable conglomerate below the eutectoid invariant. It is important to recall here that only Hbs chains are considered and not the 3D packings.

Even though homochiral sequences are slightly more favorable, the crystallization of the system leaded to the occurrence of heterochiral arrangements (racemic compound). At first glance, this behavior seems a paradox since the mostly favorable sequences are homochiral and may logically lead to the crystallization of the conglomerate. In fact, upon crystallization one of the following pathways is possible: a) the homochiral arrangement (conglomerates) b) the heterochiral arrangement (racemic compounds), and c) the random arrangement (solid solution). From the few reported cases, homochiral compositions are highly crystallogenic for conglomerate systems ${ }^{30,31}$ while for racemic compounds, the most crystallogenic compositions are near the racemic region ${ }^{29,33}$ and for solid solutions, the propensity of crystallization is independent of the composition ${ }^{32}$. These different scenarios can be schematically resumed in figure 14 by the composition evolution of crystallization temperature depending on the typology of the arrangement.
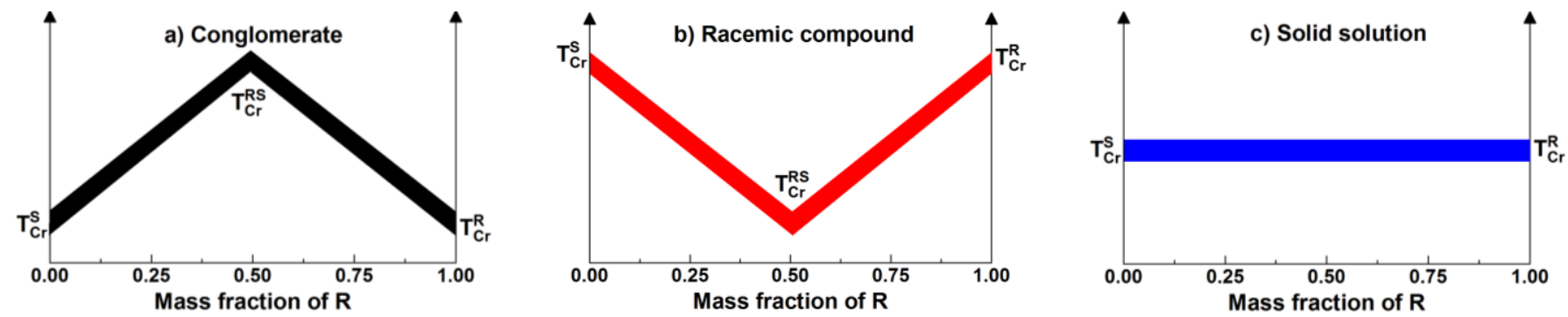

Figure 14: Schematic crystallization behavior as function of the mass fraction (idem as mole fraction) of $R$ enantiomer for a) stable conglomerate, b) stable racemic compound and c) stable solid solution After validation on a larger number of cases, this behavior could be used to screen conglomerate.

From figure $14 \mathrm{a}$ ), the crystallization of homochiral sequences near the racemic composition is kinetically unfavorable (since higher temperatures are required to crystallize the homochiral sequences at the racemic mixture compared to pure enantiomers). As displayed in figure $12 \mathrm{~b}$ ), the most energetically and kinetically advantageous pathway is the formation of heterochiral sequences (racemic compound). It seems consistent to assume that the mean free migration path achieved by an enantiomer to form homochiral sequences is longer than for heterochiral sequences since both 
enantiomers coexist in equal quantities at the racemic composition. However, for NAC-MBA the above MD simulations in the liquid state, suggest that the formation of homochiral associations are slightly more advantageous but the disadvantageous kinetic conditions for crystallization of homochiral sequences favor the occurrence of heterochiral associations. Consequently, the crystallization of the metastable racemic compound can take place.

\section{4- Conclusion}

This study aimed to assess the impact of chirality in the supercooled liquid and glassy states by evaluating the GFA, the recrystallization propensity and the relaxational landscape as function of the enantiomer ratio from a model conglomerate forming system. Moreover, an attention was paid on the structure of the hydrogen bonding in the liquid state at various ee (since the system is known to form linear HB aggregates).

As mentioned for different chiral systems, the GFA is highly influenced by chirality. For conglomerates forming systems, GFA increases with decreasing ee. Thus, the racemic mixture (ee = $0 \%$ ), is a better glass former than the pure enantiomer (ee $=100 \%$ ). Moreover, the crystallization propensity of the pure enantiomer is significantly higher compared to that of the racemic mixture. A kinetic explanation is proposed in order to illustrate this feature and considered the counter enantiomer as an impurity of the same chemical nature that can delay the crystallization process. The inverse situation was already mentioned for stable racemic compound forming systems since they are thermodynamically favored. Furthermore, crystallization from the amorphous state ineluctably leaded to the occurrence of the metastable racemic compound even though in the liquid state homochiral arrangements were slightly more favorable. From a kinetic point of view, it seems difficult for the homochiral sequences (conglomerate) to crystallize; therefore, the system evolves toward the nearest energetically state kinetically accessible corresponding to the heterochiral sequences (racemic compound). The temperature of crystallization of the amorphous chiral alloy versus e.e. might be used to spot conglomerate forming systems. Nevertheless, more cases need to be studied to validate this method.

We evidenced that the relaxational landscape of amorphous Nac-MBA is ee insensitive. Furthermore, the time scale of all the processes $\left(D, \alpha, \beta_{\mathrm{JG}}, \gamma\right)$ and the evolution of their temperature dependency are almost identical at different ee. Therefore, no significant difference in the dynamical $\mathrm{Tg}$, the fragility index $m$, the shape parameters was mentioned at variable ee. Since for all the analyzed compositions the time scale of the relaxational processes were estimated identical, while the crystallization behavior differs significantly from one composition to another. Thus, it seems that 
molecular mobility is not a key parameter in order to fully understand the crystallization behavior of chiral Nac-MBA.

One may ask if this molecular mobility insensitiveness on the ee will still survive under high pressure conditions or the existence of a hypothetical chiral discrimination in the amorphous state leading to the crystallization of the homochiral sequences. These issues will be tackled in our future works.

\section{Acknowledgements}

The authors are grateful to FEDER and Region Normandy for the financial support of this work through the FEDER MACHI project. The authors acknowledge the use of the facilities of the CRI (Villeneuve d'Ascq, France) where calculations were carried out.

\section{References}

1 I. K. Reddy and R. Mehvar, Chirality in Drug Design and Development, CRC Press, 2004.

2 J. B. Pendry, A Chiral Route to Negative Refraction, Science, 2004, 306, 1353-1355.

3 P. F. Damasceno, A. S. Karas, B. A. Schultz, M. Engel and S. C. Glotzer, Controlling Chirality of Entropic Crystals, Phys. Rev. Lett., 2015, 115, 158303.

4 C. Tschierske, Development of Structural Complexity by Liquid-Crystal Self-assembly, Angew. Chem. Int. Ed., 2013, 52, 8828-8878.

5 H. H. Wensink and G. Jackson, Generalized van der Waals theory for the twist elastic modulus and helical pitch of cholesterics, J. Chem. Phys., 2009, 130, 234911.

6 S. F. Swallen, K. L. Kearns, M. K. Mapes, Y. S. Kim, R. J. McMahon, M. D. Ediger, T. Wu, L. Yu and S. Satija, Organic Glasses with Exceptional Thermodynamic and Kinetic Stability, Science, 2007, 315, 353-356.

7 G. Coquerel and R. Tamura, in Disordered Pharmaceutical Materials, ed. rc Descamps, Wiley-VCH Verlag GmbH \& Co. KGaA, 2016, pp. 135-160.

8 L. PASTEUR, Memoires sur la relation qui peut exister entre la forme crystalline et al composition chimique, et sur la cause de la polarization rotatoire, Compt Rend, 1848, 26, 535-538.

9 I. Agranat, H. Caner and J. Caldwell, Putting chirality to work: the strategy of chiral switches, Nat. Rev. Drug Discov., 2002, 1, 753-768.

10 R. A. Sheldon, Asymmetric Catalysis in Organic Synthesis. Ryoji Noyori. John Wiley \& Sons, Chichester, 1994. xvii + 378 pp., £45.50. ISBN 0-471-57267-5, Recl. Trav. Chim. Pays-Bas, 1996, 115, 155-155.

11 A. M. Stalcup, Chiral Separations, Annu. Rev. Anal. Chem., 2010, 3, 341-363.

$12 \mathrm{~J}$. Jacques, A. Collet and S. H. Wilen, Enantiomers, racemates, and resolutions, Wiley, 1981.

13 M. Descamps and J.-F. Willart, in Disordered Pharmaceutical Materials, Wiley-Blackwell, 2016, pp. $1-56$.

14 M. Rams-Baron, R. Jachowicz, E. Boldyreva, D. Zhou, W. Jamroz and M. Paluch, in Amorphous Drugs: Benefits and Challenges, eds. M. Rams-Baron, R. Jachowicz, E. Boldyreva, D. Zhou, W. Jamroz and M. Paluch, Springer International Publishing, Cham, 2018, pp. 1-7.

15 S. Onoue, H. Sato, K. Ogawa, Y. Kawabata, T. Mizumoto, K. Yuminoki, N. Hashimoto and S. Yamada, Improved dissolution and pharmacokinetic behavior of cyclosporine A using high-energy amorphous solid dispersion approach, Int. J. Pharm., 2010, 399, 94-101. 
16 M. Newa, K. H. Bhandari, J. O. Kim, J. S. Im, J. A. Kim, B. K. Yoo, J. S. Woo, H. G. Choi and C. S. Yong, Enhancement of Solubility, Dissolution and Bioavailability of Ibuprofen in Solid Dispersion Systems, Chem. Pharm. Bull. (Tokyo), 2008, 56, 569-574.

17 M. T. Ottou Abe, N. T. Correia, L.-C. Valdes, J. M. B. Ndjaka and F. Affouard, Local molecular organizations of ibuprofen, flurbiprofen and ketoprofen in the liquid phase: Insights from molecular dynamics simulations, J. Mol. Liq., 2015, 205, 74-77.

18 A. R. Brás, J. P. Noronha, A. M. M. Antunes, M. M. Cardoso, A. Schönhals, F. Affouard, M. Dionísio and N. T. Correia, Molecular Motions in Amorphous Ibuprofen As Studied by Broadband Dielectric Spectroscopy, J. Phys. Chem. B, 2008, 112, 11087-11099.

19 P. J. Marsac, H. Konno and L. S. Taylor, A Comparison of the Physical Stability of Amorphous Felodipine and Nifedipine Systems, Pharm. Res., 2006, 23, 2306-2316.

20 Y. R. Roos and M. A. Karel, Crystallization of Amorphous Lactose, J. Food Sci., 1992, 57, 775-777.

21 T. Cordeiro, C. Castiñeira, D. Mendes, F. Danède, J. Sotomayor, I. M. Fonseca, M. Gomes da Silva, A. Paiva, S. Barreiros, M. M. Cardoso, M. T. Viciosa, N. T. Correia and M. Dionisio, Stabilizing Unstable Amorphous Menthol through Inclusion in Mesoporous Silica Hosts, Mol. Pharm., 2017, 14, 3164-3177.

22 S. J. Schmidt and A. M. Lammert, Physical Aging of Maltose Glasses, J. Food Sci., 1996, 61, 870 875.

$23 \mathrm{~S}$. Shirke and R. D. Ludescher, Molecular mobility and the glass transition in amorphous glucose, maltose, and maltotriose, Carbohydr. Res., 2005, 340, 2654-2660.

24 B. C. Hancock and S. L. Shamblin, Molecular mobility of amorphous pharmaceuticals determined using differential scanning calorimetry, Thermochim. Acta, 2001, 380, 95-107.

$25 \mathrm{~J}$. Sibik and J. A. Zeitler, Direct measurement of molecular mobility and crystallisation of amorphous pharmaceuticals using terahertz spectroscopy, Adv. Drug Deliv. Rev., 2016, 100, 147157.

26 K. Adrjanowicz, K. Kaminski, M. Paluch and K. Niss, Crystallization Behavior and Relaxation Dynamics of Supercooled S-Ketoprofen and the Racemic Mixture along an Isochrone, Cryst. Growth Des., 2015, 15, 3257-3263.

27 Q. Viel, L. Delbreilh, G. Coquerel, S. Petit and E. Dargent, Molecular Mobility of an Amorphous Chiral Pharmaceutical Compound: Impact of Chirality and Chemical Purity, J. Phys. Chem. B, 2017, 121, 7729-7740.

28 M. T. O. Abe, M. T. Viciosa, N. T. Correia and F. Affouard, Impact of chirality on peculiar ibuprofen molecular dynamics: hydrogen bonding organization and syn vs. anti carboxylic group conformations, Phys. Chem. Chem. Phys., 2018, 20, 29528-29538.

29 K. Adrjanowicz, K. Kaminski, M. Tarnacka, K. Szutkowski, L. Popenda, G. Bartkowiak and M. Paluch, The effect of hydrogen bonding propensity and enantiomeric composition on the dynamics of supercooled ketoprofen - dielectric, rheological and NMR studies, Phys. Chem. Chem. Phys., 2016, 18, 10585-10593.

30 B. Atawa, N. Couvrat, G. Coquerel, E. Dargent and A. Saiter, Impact of chirality on the Glass Forming Ability and the crystallization from the amorphous state of 5-ethyl-5-methylhydantoin, a chiral poor glass former, Int. J. Pharm., 2018, 540, 11-21.

31 B. Atawa, N. Couvrat, G. Coquerel, E. Dargent and A. Saiter, Chirality impact on physical ageing: An original case of a small organic molecule, Mater. Lett., 2018, 228, 141-144.

32 H. E. Gallis, J. C. van Miltenburg and H. A. J. Oonk, Polymorphism of mixtures of enantiomers: A thermodynamic study of mixtures of D- and L-limonene, Phys. Chem. Chem. Phys., 2000, 2, 56195623.

33 Q. Viel, C. Brandel, Y. Cartigny, M. E. S. Eusébio, J. Canotilho, V. Dupray, E. Dargent, G. Coquerel and S. Petit, Crystallization from the Amorphous State of a Pharmaceutical Compound: Impact of Chirality and Chemical Purity, Cryst. Growth Des., 2017, 17, 337-346.

$34 \mathrm{E}$. Dachwitz and M. Stockhausen, On the Occurance of Stereoisomeric Effects in the Dielectric Relaxation Behaviour of Pure Liquid, Z. Für Naturforschung A, 2014, 43, 476-480. 
35 B. Atawa, N. T. Correia, N. Couvrat, F. Affouard, G. Coquerel, E. Dargent and A. Saiter, Molecular mobility of amorphous $\mathrm{N}$-acetyl- $\alpha$-methylbenzylamine and Debye relaxation evidenced by dielectric relaxation spectroscopy and molecular dynamics simulations, Phys. Chem. Chem. Phys., 2019, 21, 702-717.

36 THE DL POLY 2 USER MANUAL - PDF, http://docplayer.net/14320255-The-dl-poly-2-usermanual.html, (accessed May 31, 2018).

37 J. Wang, R. M. Wolf, J. W. Caldwell, P. A. Kollman and D. A. Case, Development and testing of a general amber force field, J. Comput. Chem., 2004, 25, 1157-1174.

38 S. Druot, M. N. Petit, S. Petit, G. Coquerel and N. B. Chanh, Experimental Data and Modelling of the Interactions in Solid State and in Solution between (R) and (S) N-Acetyl- $\alpha$ - Methylbenzylamine. Influence on Resolution by Preferential Crystallization, Mol. Cryst. Liq. Cryst. Sci. Technol. Sect. Mol. Cryst. Liq. Cryst., 1996, 275, 271-291.

39 X. Monnier, A. Saiter and E. Dargent, Vitrification of PLA by fast scanning calorimetry: Towards unique glass above critical cooling rate?, Thermochim. Acta, 2017, 658, 47-54.

40 O. Wallach, Zur Kenntniss der Terpene und der ätherischen Oele, Justus Liebigs Ann. Chem., 1895, 286, 90-118.

41 K. L. Ngai, An extended coupling model description of the evolution of dynamics with time in supercooled liquids and ionic conductors, J. Phys. Condens. Matter, 2003, 15, S1107-S1125.

42 C. J. F. Böttcher, in Theory of Electric Polarization (Second Edition), ed. C. J. F. Böttcher, Elsevier, Amsterdam, 1973, pp. 9-58.

43 S. Bauer, H. Wittkamp, S. Schildmann, M. Frey, W. Hiller, T. Hecksher, N. B. Olsen, C. Gainaru and R. Böhmer, Broadband dynamics in neat 4-methyl-3-heptanol and in mixtures with 2-ethyl-1hexanol, J. Chem. Phys., 2013, 139, 134503.

44 M. Stockhausen and E. Dachwitz, Evidence of stereoisomeric effects in dielectric relaxation, Chem. Phys. Lett., 1985, 121, 77-79.

$45 \mathrm{E}$. Dachwitz and M. Stockhausen, On the Occurance of Stereoisomeric Effects in the Dielectric Relaxation Behaviour of Pure Liquid, Z. Für Naturforschung A, 1988, 43, 476-480.

46 D.-M. Shin, Y.-H. Hwang, J.-H. Ko and S. Kojima, Relaxation behaviors of enantiomorphic Sibuprofen as revealed by dielectric and photon correlation spectroscopies, Curr. Appl. Phys., 2015, 15, 958-963.

47 C. C. Mjojo and H. K. Welsh, Dielectric studies of orientational disorder in D- and L-systems of camphor and camphoric anhydride, J. Chem. Soc. Faraday Trans., 1992, 88, 2909-2913.

48 A. Lerbret, P. Bordat, F. Affouard, M. Descamps and F. Migliardo, How Homogeneous Are the Trehalose, Maltose, and Sucrose Water Solutions? An Insight from Molecular Dynamics Simulations, J. Phys. Chem. B, 2005, 109, 11046-11057.

$49 \mathrm{I}$. Alkorta and J. Elguero, Self-discrimination of enantiomers in hydrogen-bonded dimers, J. Am. Chem. Soc., 2002, 124, 1488-1493.

50 R. Hemmati and K. Patkowski, Ab Initio Study of Chiral Discrimination in the Glycidol Dimer, J. Phys. Chem. A, 2020, 124, 9436-9450.

51 S. Portmann, A. Inauen, H. P. Lüthi and S. Leutwyler, Chiral discrimination in hydrogen-bonded complexes, J. Chem. Phys., 2000, 113, 9577-9585. 